I sHould like to comment on the article 'Advances in Chinese Restaurant Research' (News and Views 267, 756; 1977). Unfortunately your anonymous correspondent seems unaware of the background to a field which may have some superficially amusing characteristics but, in fact concerns some potentially serious issues.

The topic of the Chinese Restaurant Syndrome is hardly 'gripping' the Boston Medical Research Community. In fact, although it mildly interests most people, it 'grips' only those who get fairly severe symptoms. In trying to point out that the name of this Syndrome was unfortunate (since glutamate is used in other types of restaurants), I gave as examples, Japanese, Italian and French; I did not feel that I had to enumerate the names of all types of cuisine available in the Boston area. 'All-American' establishments are by no means 'blameless'; if by AllAmerican food one means steaks, for instance, it has been found in some establishments that an $8 \mathrm{oz}$ steak is not unlikely to be 'doctored' with $0.9 \mathrm{~g}$ of monosodium glutamate. As for the listing of what the correspondent found to be a disagreeably large number of symptoms, I will quote, by comparison, a recent description of yet another illdefined, but quite real human malady: Symptoms of "Classic migraine . . .in addition to the head pain ... may also include photophobia, nausea, vomiting, constipation or diarrhea, weight gain and fluid retention followed by diuresis, scotomas or visual field defects, paresthesias or defects in motility, vertigo, and elevation of blood pressure.' (from Dalessio Dietary Migraine, Am. Family Phys. 6 No. 6, 60; 1972).

A large literature exists on the effects of glutamate in both animals and humans. An interest in the adverse effects, to (presumably) glutamate, both in animals and in some humans, has existed for a number of years. It is fairly well established that parenteral

\section{Chinese Restaurant Syndrome}

administration of glutamate causes vomiting in humans. Various studies, primarily since 1968, have indicated that the so-called Chinese Restaurant Syndrome is probably caused by the raised levels of free glutamate that are frequently added to such foods. This general problem is of both theoretical and practical interest. Many people (including many physicians) are unaware of so-called Chinese Restaurant Syndrome; people with fairly severe reactions to what is presumably monosodium glutamate, have been unnecessarily caused extended periods of anxiety (thinking that they had some neurological problem) because of this lack of general knowledge. A number of young children have been subjected to extensive neurological testing and have apparently been misdiagnosed and incorrectly treated because of a lack of awareness of 'glutamate overload' intolerance. (Reif-Lehrer \& M. G. Stemmermann New Engl. J. Med. 293, 1204; 1975; Reif-Lehrer, Pediatrics 58, 771; 1976.) There is also an increasing literature on glutamic acid as a neuroexcitatory substance and this amino acid is currently being examined as a putative neurotransmitter in certain cells. $\mathrm{Nu}$ merous investigators have reported that young rodents treated with glutamate are subject to both retinal and hypothalamic damage. Similar results have been obtained in the hypothalamus of monkeys, but are more controversial.

Animals which have been examined do respond to glutamate overloading with convulsions. A variety of other responses in animals have also been reported, and either glutamate or aspartate are routinely used to eliminate the $B$ wave of the electroretinogram of isolated retinas, again indicating damage to the cells of the inner retina. Several studies in humans, as well as animals, have shown that ingestion of raised levels of free glutamate does indeed lead to abnormally high blood levels of this amino acid.

In the course of some studies concerned with glutamate metabolism in the retina, I became interested in the more general topic of glutamate metabolism in humans and in particular in a question which had apparently remained unanswered in the literature: namely, how many people experience the strange phenomenon called Chinese Restaurant Syndrome, and what may be different about the metabolism of these sensitive individuals. The fact that this issue is of importance to others may be attested to by public hearings (July 25-26) before the FASEB Select Committee on GRAS Substances on Glutamates, which were held in Washington. A final report of this Committee should be ready early in 1978. The opinion expressed in the tentative FASEB report and confirmed at the Washington hearings was, that the issues concerning glutamate consumption are of importance and need further exploration. Such further exploration will of course, require time, money, appropriate expertise, and an interest on the part of the scientific community to explore the question without preconceived bias.

LiAne ReIf-LeHRER

Department of Ophthalmology,

Harvard Medical School, and

Department of Retina Research,

Eye Research Institute of Retina

Foundation

Our correspondent replies: I am very sorry that Dr Reif-Lehrer mistook my light-hearted comments for an attack on research into the harmful effects of glutamate. What I did intend to question was the usefulness of accumulating a vast body of anecdotal reports, when they are not accompanied by parallel tests for the substance in question. This kind of approach makes it all too easy for the food industry to cry "not proven'.

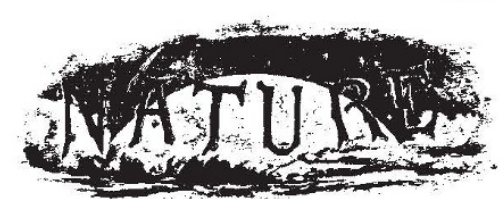

\section{A hundred years ago}

From the last report of Dr. Dohrn, the director, we notice that the zoologica! station at Naples has daveloped a most remarkable degree of activity, and is becoming a valuable centre of biological research. By the generosity of the Prussian Government it has been provided with a small steamer, and the uninterrupted expeditions in this vessel have secured to the laboratcries an enormous and most varied stock of material for research. Dr. Dohrn has carcfully organised a plan for the systematic examination of the entire fauna of this part of the sea, to be accompanied by exhaustive description. The literary portion of the work will consist of elaborate monographs on all the families and species represented in the Gulf of Naples. They will not be prepared by the members of the station only, but it is hoped to procure the assistance of all familiar with this special department, and the contributions can be in English, French, German, or Italian. Two monographs on the Elenophorae and Balanoglossi will appear during the present year, and arrangements have been made for the speedy preparation of eleven others.

THE Radicals in the French Chamber cannot be accused of opposition to the claims of science. We notice that in a late session a member of the extreme left proposed an amendment to the budget of instruction, which provided for the appropriation of 30,000 francs for an expedition to California to observe the next transit of Mercury, 40,000 for the continuation of the explorations in Northern Africa, where it has been proposed to admit water from the Mediterranean, and 100,000 to enable the $\mathrm{Abb}$ Debes to make a journey across Africa from Zanzibar to the Congo. As the appropriation was granted, we may hope soon to see the latter portion of it cause the appearance of a new rival of Stanley for the Abbe has had, like Livingstone, invaluable experiences as a missionary, which will enable him to enter upon the undertaking with great promises of success.

From Nature 17, 21 Fcb, 329; 1878 